\title{
Longitudinal evaluation of an mHealth overweight and obesity management tool
}

\author{
Ida Olivia Juhl Langkjær ${ }^{1} \wedge$, Cilius Esmann Fonvig ${ }^{2,3}$, Louise Aas Holm ${ }^{2} \wedge$, Andreas Friis Pihl ${ }^{4,5}$, \\ Jens-Christian Holm ${ }^{1,2,3} \wedge$
}

${ }^{1}$ University of Copenhagen, Faculty of Health and Medical Sciences, Copenhagen, Denmark; ${ }^{2}$ Faculty of Health and Medical Sciences, Novo Nordisk Foundation Center for Basic Metabolic Research, University of Copenhagen, Copenhagen, Denmark; ${ }^{3}$ Dr Holm App Aps., Holbæk, Denmark; ${ }^{4}$ Research Unit of General Practice, University of Southern Denmark, Odense, Denmark; ${ }^{5}$ Roche Diagnostics Denmark, Hvidovre, Denmark

Contributions: (I) Conception and design: CE Fonvig, JC Holm; (II) Administrative support: None; (III) Provision of study materials or patients: CE Fonvig, JC Holm; (IV) Collection and assembly of data: CE Fonvig; (V) Data analysis and interpretation: All authors; (VI) Manuscript writing: All authors; (VII) Final approval of manuscript: All authors.

Correspondence to: Jens-Christian Holm. Kalundborgvej 114, 4300 Holbæk, Denmark. Email: jch@drholm.com.

\begin{abstract}
Background: Efficient obesity treatment protocols are lacking. This study reports treatment results from a web-based application, originally developed for use in an in-person healthcare setting providing health, overweight, and obesity management.

Methods: The web application DrHolmApp (WADHA) was evaluated in adult users two years after it was launched. The WADHA provides a personal and tailored treatment plan comprising a series of detailed action advices on everyday life, constructed from the user's input to a thorough online questionnaire. Throughout the subscription period, the WADHA users have full access to online healthcare professional support. We conducted a longitudinal cohort study using self-reported data.

Results: This study included 940 adult WADHA users (861 female). The median body mass index (BMI) change across all WADHA users was -0.63 BMI points (95\% CI: -0.7 to $-0.57, \mathrm{P}<0.001) .665$ (71\%) of all WADHA users reduced their BMI (median reduction: $0.94,95 \%$ CI: 0.88 to 1.02 ). In the subset with obesity $(\mathrm{n}=675), \mathrm{BMI}$ was reduced in $72 \%$. The median number of days per week with physical activity for at least one hour per day increased with 1.5 days per week (from 2 days per week at baseline, $\mathrm{P}<0.001$ ). Subsequently, the WADHA users improved their mood, quality of life, and body image satisfaction and reduced their appetite, bullying, and wish for weight loss (all $\mathrm{P}<0.001$ ). A higher number of consultations associated with greater weight loss $(\mathrm{P}<0.001)$ independent of age and degree of obesity at treatment initiation.

Conclusions: Seventy-one percent of the WADHA users experienced weight loss, concomitant to an increased level of physical activity, improved mood, quality of life, and body image satisfaction, and reduced appetite, degree of bullying, and wish for weight loss.
\end{abstract}

Keywords: Body mass index (BMI); mobile health (mHealth); obesity; treatment; weight loss

Received: 10 March 2021; Accepted: 24 June 2021; Published: 20 January 2022.

doi: $10.21037 /$ mhealth-21-8

View this article at: https://dx.doi.org/10.21037/mhealth-21-8

\footnotetext{
^ ORCID: Ida Olivia Juhl Langkjær, 0000-0001-7647-3080; Cilius Esmann Fonvig, 0000-0002-5031-0125; Louise Aas Holm, 0000-00025439-5429; Andreas Friis Pihl, 0000-0003-0158-7205; Jens-Christian Holm, 0000-0003-4653-342X.
} 


\section{Introduction}

Worldwide, obesity is one of the largest public health challenges of this century. In 2016, the number of adults living with overweight reached 1.9 billion, which corresponds to $39 \%$ of the global adult population, of whom 650 million exhibited obesity (1). If the current development continues, by the year of $2030,58 \%$ of the global adult population will live with overweight of which $20 \%$ will suffer from obesity (2).

Obesity causes multiple complications. Among the most common complications are hypertension, fatty liver, dyslipidemia, sleep apnea, and depression alongside high prevalence of concomitant type 2 diabetes mellitus, cardiovascular diseases, and cancers. Furthermore, overweight and obesity are found to be associated with reduced physical activity, increased inactivity, poor sleep, and decreased quality of life (3). Therefore, development of innovative and effective methods to counter obesity and its related complications worldwide is needed.

In general, the market for health applications, including applications focusing on weight loss, is expanding, but studies of weight loss applications have shown a significant lack of expert involvement and a lack of evidence-based treatment methods (4-6).

With the growing market and increasing interest from the population, healthcare professionals need to consider implementing mHealth in overweight and obesity treatment strategies. As an example, patients in Germany now get reimbursed when general practitioners prescribe an app in regards of treatment, and in Great Britain they have established their National Health Service Apps Library as well as strategic efforts ensuring of implementation into the clinical practices. Despite these examples, guidelines regarding the use of mHealth in overweight and obesity treatment and criteria for standardized application evaluations, are lacking (6).

\section{The Holbaek obesity treatment (HOT) protocol}

The Web Application DrHolmApp (WADHA) is developed according to the HOT protocol, which is an evidencebased, multifaceted obesity treatment protocol designed for the management of overweight and obesity in the pediatric population (7). The treatment is based on general recommendations combined with the perspective and insights of obesity understood as a disease and physiological insights into the endocrine regulation of fat mass; especially how the body adapts when challenged by weight loss $(8,9)$. These insights include an understanding of the body shifting into an energy-preserving mode when the individual is initiating fat mass reducing actions, such as a reduced caloric intake or an increased level of physical activity. The HOT protocol has induced weight loss in $65-85 \%$ of more than 3,000 patients $(7,10)$-with reductions in the degree of obesity independent of baseline degree of adiposity, age, social class (7), glucose metabolism (11), genetic risk score (12), and disturbed eating (13)—reductions in parents' body mass index (BMI) (14), and amelioration of obesityrelated complications in children and adolescents $(7,15)$. This includes reductions in blood pressure (16-18), blood lipid concentrations $(19,20)$, fat accumulation in liver and skeletal muscle (21), and obstructive sleep apnea (22), and improvements in quality of life $(23,24)$ and psychosocial well-being (24).

The HOT protocol has been implemented in hospital settings and the primary healthcare sector in more than 75 out of 98 municipalities in Denmark $(7,10,15)$.

The HOT protocol with its questionnaires and treatment plans have been converted into an online obesity treatment tool, the WADHA.

Here we present the first results of the digital transformation of the HOT protocol from an out-patient treatment clinic to an online web solution.

We present the following article in accordance with the STROBE reporting checklist (available at https://dx.doi. org/10.21037/mhealth-21-8).

\section{Methods}

This longitudinal questionnaire-based survey study recruited users of an online web application from December 2017 to December 2019.

\section{WADHA}

The WADHA is developed by healthcare professional obesity specialists and is a responsive homepage that mimics a smartphone application on all mobile devices or laptops. By subscribing, the WADHA user gets access to a tailormade and individualized treatment plan, a multimedia library with text and video clips in obesity education, a grocery shopping guide, healthy recipes, a diary, graphical treatment trajectories, inspiration to physical activity, and online healthcare professional support. The personal treatment plan is constructed automatically after each 
Table 1 The subjective visual analogue scale score assessment

\begin{tabular}{|c|c|c|}
\hline Domains & Definition & Question \\
\hline Appetite & The degree of feeling hungry & $\begin{array}{l}\text { "Are you very hungry [10], not hungry [0], or } \\
\text { somewhere in between?" }\end{array}$ \\
\hline Bullying & $\begin{array}{l}\text { Being socially excluded, teased, beaten, or bothered } \\
\text { in a repulsive manner }\end{array}$ & $\begin{array}{l}\text { "Are you bullied a lot [10], never bullied [0], or } \\
\text { somewhere in between?" }\end{array}$ \\
\hline Motivation & The degree of the wish to lose weight & $\begin{array}{l}\text { "Do you want to lose weight [10], do you not care to } \\
\text { lose weight [0], or somewhere in between?" }\end{array}$ \\
\hline Body Image Satisfaction & $\begin{array}{l}\text { The perception of own body image and contentment } \\
\text { of own physical appearance }\end{array}$ & $\begin{array}{l}\text { "Are you happy with your body [10], are you } \\
\text { dissatisfied with your body [0], or somewhere in } \\
\text { between?" }\end{array}$ \\
\hline
\end{tabular}

Table 1 is reprinted by permission from Springer Nature Customer Service Centre GmbH: Springer Nature, Quality of Life Research, Subjective evaluation of psychosocial well-being in children and youths with overweight or obesity: the impact of multidisciplinary obesity, Cilius Esmann Fonvig et al., 2017.

online consultation by an algorithm based on the answers made in the consultation. The online consultations consist of 90 questions that thoroughly evaluate important details and aim to identify unfavorable logistics and choices in daily life in order to control the environment and thus manage the degree of overweight and related complications. These include questions regarding quantities, frequencies, ingredients, and qualities on meals, eating patterns, appetite, intake of foods high in sugar and fat, physical activity, inactivity, sleep, alcohol and smoking habits, means of transportation, among other. The individualized treatment plan accommodates several strategies, including a meal eating exercise to be used at dinner to help control appetite and meal patterns. The WADHA user is prompted an answer for each question, which is then used to construct a treatment plan that is designed to tailor-make changes in daily life habits (Appendix 1). Furthermore, many of the 90 questions also serve as indirect or direct contributors to the WADHA users' understanding and awareness of obesity, regardless of whether or not a question results in an actual item on the treatment plan.

The online consultation also evaluates psychosocial well-being using a six-item subjective assessment of the domains: mood, quality of life, appetite, bullying, wish for weight loss, and body image satisfaction based on the WADHA user's general everyday life during the past few weeks. This is assessed by use of a visual analogue scale
(VAS) score used in treatment and studies of childhood and adolescent obesity. The assessment tool was developed for daily clinical practice, comprising simple questions addressing each of these six important psychosocial qualities in an understandable manner, where the WADHA users are asked to rate the abovementioned parameters on a VAS score from 0 to 10 as described by Fonvig et al. (24). As an example, the WADHA users are asked "Do you have the best life [10], the worst life [0], or somewhere in between?" when asked about their quality of life (Table 1). Based on the WADHA user's perception of the specific parameter, the user places the curser on a blank line marked with 0 and 10 in each end. The exact value is calculated by the software.

The online consultation is primarily digital with an option to receive healthcare provided support. The online consultations consist of the questionnaire described above with the personal treatment plan as the immediate outcome.

Follow-up consist of repetitions of the online consultation and can be repeated an unlimited number of times; the only limitation is that the next online consultation is locked for 14 days after each consultation. The overall goal is to periodically adjust and revise the treatment plan according to new habits and relevant challenges in daily life and thereby process-develop the mind of the WADHA user to continuously cope and comply with the treatment plan which aims to control the environment amidst individual challenges. 
The WADHA is publicly available on the internet $(25,26)$. Users can buy a subscription for 3, 12, or 24 months.

None of the WADHA users received any compensations, reimbursements, or payments for participating in the program.

All assessments are self-reported by the WADHA users in each of the online consultations. The WADHA users were consecutively included by subscription over time and completed a varying number of online consultations. The present study only reports the first and the last consultation regardless of the individual WADHA user had two or more than 30 consultations. Follow-up is therefore the latest consultation before data evaluation. Data extraction took place two years after launching the WADHA.

The WADHA is currently available in Danish, English, Arabic, and Norwegian, although only data from the Danish version has been evaluated in the present study.

\section{Ethics}

All included data from the study population is given with consent, and WADHA users have consented to having their data analyzed and stored according to the European GDPR regulations (27).

This study was conducted according to the Helsinki Declaration (as revised in 2013). In accordance with Danish Law [section 14(2) of the Committee Act of the Danish National Committee on Health Research Ethics], this questionnaire-based survey study, which does not involve human biological material, should not be notified or reported to a research ethics committee.

\section{Statistical analysis}

Normality of data was evaluated using histograms and Q-Q plots. All variables, except from age, exhibited a non-normal distribution. The paired Wilcoxon signed rank test was used to analyze differences in continuous variables (age, height, weight, BMI, treatment duration, number of consultations, number of days per week with physical activity (PA) more than 1 hour/day, and the domains on the VAS) from baseline to follow-up and the corresponding nonparametric CI. The sample estimates were expressed as a '(pseudo) median' difference. Skewness of all continuous variables was evaluated by residual plots, where random patterns identified variables with no need for data transformation. All the variables showed no need for data transformation. Accordingly, applying the 'skewness' measure (R-package: 'moments') showed that none of the variables exhibited a skewness number closer to 0 (zero) after log-transformation. The Chi-squared test was used to perform between-group analysis of gender composition.

Associations with change in BMI were investigated by multivariate linear regression models adjusted for age, gender, baseline degree of obesity, treatment duration, and number of online consultations.

The level of significance was set at $\mathrm{P}<0.05$. Statistical analyses were performed using " $\mathrm{R}$ " statistical software version 4.0.2 (28).

\section{Results}

\section{Study sample}

This study included 979 adult WADHA users who had completed at least two online consultations.

Exclusion criteria were (I) a height change of more than $9 \mathrm{~cm}$ during treatment, (II) a weight change of more than an average of $1.3 \mathrm{~kg}$ per day during the course of treatment, and (III) a BMI-change of more than an average of 0.5 points per day during the course of treatment. Thirty-nine were excluded due to reported abnormal changes in height $(\mathrm{n}=36)$, weight $(n=3)$, or BMI $(n=0)$.

\section{WADHA user characteristic developments}

Among the remaining 940 (861 female) WADHA users, the median $\mathrm{BMI}$ at follow-up was 33.3 [interquartile range (IQR): 29.0-37.9] (33.9 at baseline). At follow-up, the fraction with normal weight had increased to $4.0 \%$ (from $2.3 \%$ ), the fraction with overweight had increased to $28.5 \%$ (from $25.9 \%$ ), and the fraction with obesity had decreased accordingly (see Table 2).

The median treatment duration was 52 days (IQR: 25-84) and the median number of consultations was 3 (IQR: 2-4) ranging up to more than 40 consultations.

The median VAS scores at follow-up were: $\operatorname{mood} 7.1$ (6.7 at baseline), quality of life 7.3 (6.9 at baseline), appetite 6.2 (6.7 at baseline), bullying 0.0 (0.1 at baseline), wish for weight loss 10.0 (10.0 at baseline), body image satisfaction 2.3 (1.5 at baseline).

\section{Effectiveness on weight loss}

The median BMI change of all 940 WADHA users was $-0.63 \mathrm{BMI}$ points (95\% CI: -0.70 to $-0.57, \mathrm{P}<0.001$ ). A 
Table 2 Characteristics of the 940 WADHA users

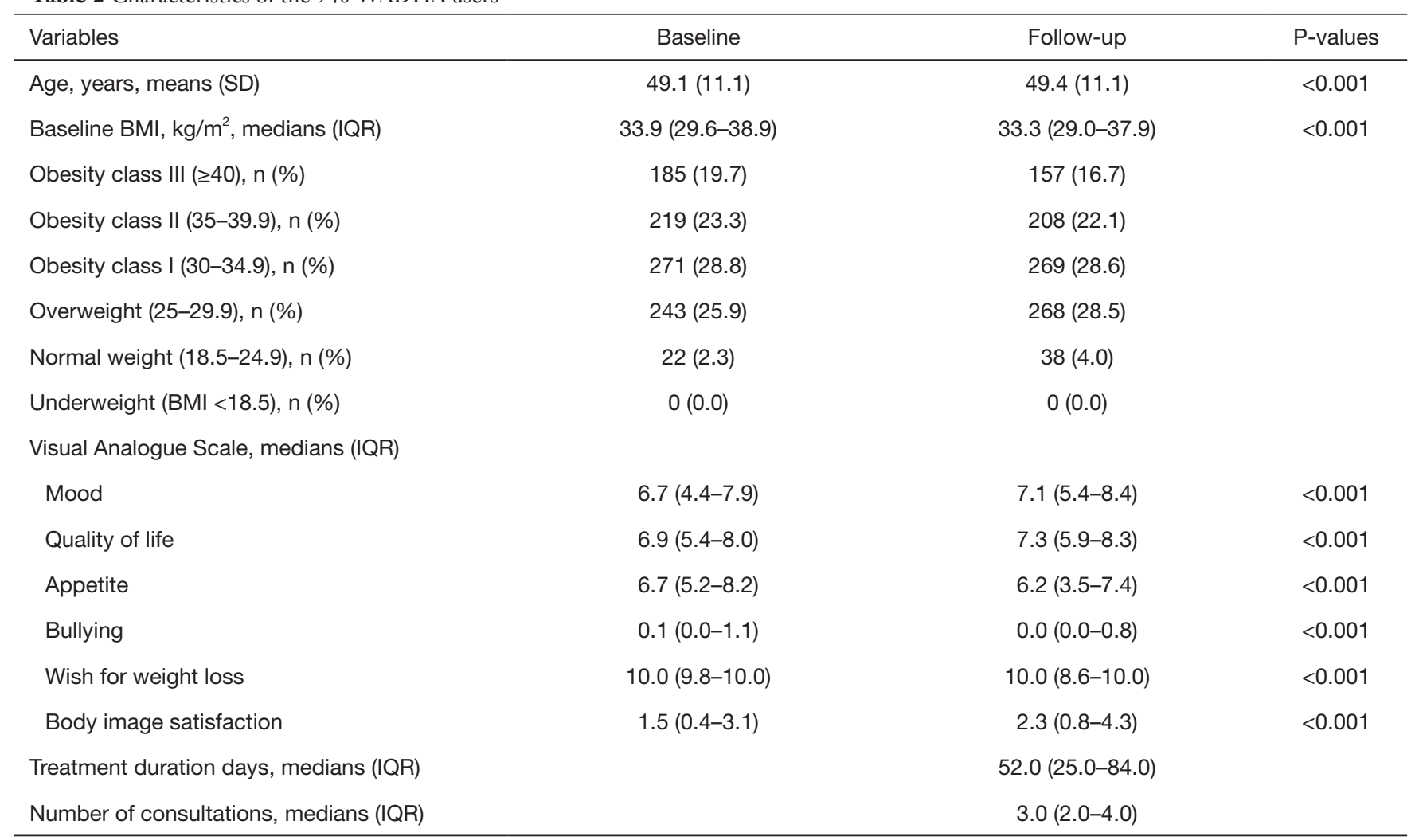

WADHA, Web Application DrHolmApp; SD, standard deviation; BMI, body mass index; IQR, interquartile range.

Table 3 Multiple linear regression showing the relationship between the change in BMI and age, gender, and baseline BMI in the 940 adult WADHA user

\begin{tabular}{|c|c|c|c|}
\hline \multirow{2}{*}{ Independent variables ( $\triangle \mathrm{BMI}$ as the dependent variable) } & \multicolumn{3}{|c|}{ Basic model } \\
\hline & $\beta$ (SE) & $\mathrm{R}^{2}$ & $P$ \\
\hline Age, years & $-0.02(0.004)$ & 0.01 & $<0.001$ \\
\hline Gender & $-0.34(0.17)$ & 0.02 & 0.04 \\
\hline $\mathrm{BMI}$ at baseline & $-0.02(0.01)$ & 0.02 & 0.01 \\
\hline
\end{tabular}

Estimates $(\beta)$, standard errors (SE), and correlation coefficients $\left(R^{2}\right)$ of the relationship between the change in BMI, age, gender, and baseline BMI. WADHA, Web Application DrHolmApp; BMI, body mass index.

total of $641(71 \%)$ of the WADHA users reduced their BMI (median change: $-0.94,95 \% \mathrm{CI}:-1.02$ to -0.88 ), while $9 \%$ maintained their BMI, and $20 \%$ increased their BMI (median increase: $0.68,95 \%$ CI: $0.57-0.80$ ).

The median number of days per week with PA for at least one hour per day increased with 1.5 days per week $(95 \%$ CI: $1.5-1.5, \mathrm{P}<0.001$ ) from 2.0 days per week at baseline.

Subsequently, the WADHA users improved their mood by 0.4 points $(\mathrm{P}<0.001)$, quality of life by 0.4 points $(\mathrm{P}<0.001)$, and body image satisfaction by 0.8 points $(\mathrm{P}<0.001)$. They reduced their appetite by 0.7 points $(\mathrm{P}<0.001)$, bullying by 0.2 points $(\mathrm{P}<0.001)$, and the wish for weight loss by 0.8 points $(\mathrm{P}<0.001)$.

In the subset of WADHA users with obesity $(\mathrm{n}=675)$, $\mathrm{BMI}$ was reduced in $72 \%$, while $8 \%$ had an unchanged BMI, and $20 \%$ increased BMI. 
Table 4 Multiple linear regression showing the relationship between the change in BMI and age, gender, and baseline BMI (like in Table 3) and further adjusted for treatment duration (model A) and number of consultations (model B) in the 940 adult WADHA users

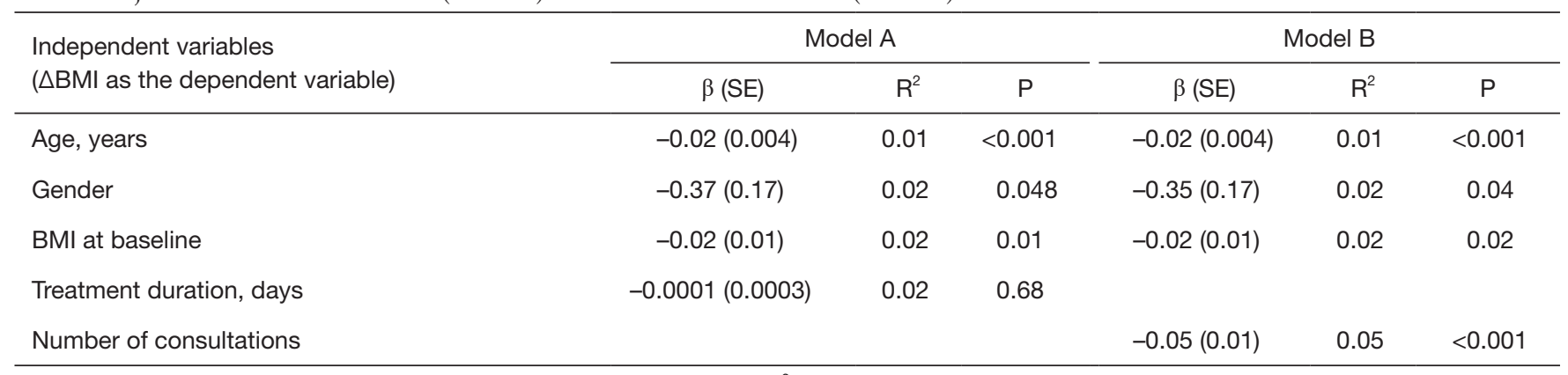

Estimates $(\beta)$, standard errors (SE), and correlation coefficients $\left(R^{2}\right)$ of the relationship between the change in BMI and age, gender, baseline BMI, and further adjusted for treatment duration (model A) and number of consultations (model B). WADHA, Web Application DrHolmApp; BMI, body mass index.

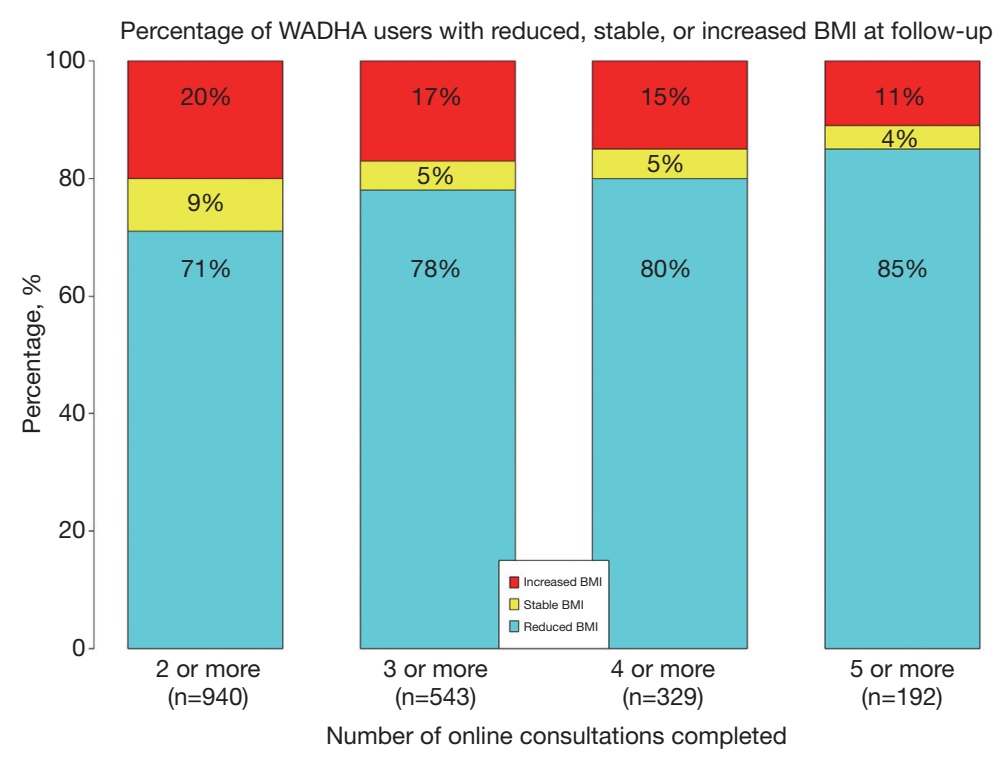

Figure 1 The fraction of WADHA users with a reduced, stable, or increased BMI at follow-up; categorized by number of completed online consultations. The left column shows all the 940 WADHA users who completed at least 2 online consultations of whom $71 \%$ reduced their BMI, the second column from the left shows the 543 WADHA users who completed at least 3 online consultations of whom $78 \%$ reduced their BMI, etc. WADHA, Web Application DrHolmApp; BMI, body mass index.

\section{Associations with weight loss}

In the basic linear regression model (Table 3), the change in BMI was inversely associated with age $(\mathrm{P}<0.001)$ and gender $(\mathrm{P}=0.04)$, and the $\mathrm{BMI}$ at baseline $(\mathrm{P}=0.01)$, indicating that higher age, the male gender, and a higher $\mathrm{BMI}$ at baseline associate with greater reductions in BMI.

When further adjusting for either treatment duration $(\mathrm{P}=0.68$; Table 4 , model $\mathrm{A})$ or number of consultations $(\mathrm{P}<0.001$; Table 4, model $\mathrm{B})$ in each their model, only number of consultations was associated (inversely) with the change in BMI. This indicates that a higher number of consultations associate with greater reductions in BMI.

Accordingly, a stepwise categorization of the WADHA users, based on the number of online consultations completed, showed that higher fractions reduce their BMI with higher numbers of completed consultations (Figure 1). In another regression analysis, adjusted for age, gender, baseline BMI, number of days per week with PA for at least one hour per day at baseline, and number of consultations, the change in $\mathrm{PA}$ was 
inversely associated with the change in $\mathrm{BMI}(\mathrm{P}<0.001)$.

In addition to the 940 WADHA users who completed at least two online consultations, another 760 (652 female, median age $=48.0$ years, median $\mathrm{BMI}=33.2$ ) adult WADHA users completed just one online consultation (WADHA noFollow-up ). The WADHA noFollow-up $_{\text {users were }}$ comparable in age (48.9 vs. 48.0 years, $\mathrm{P}=0.06$ ) and exhibited a slightly lower baseline $\mathrm{BMI}(33.2$ vs. $33.9, \mathrm{P}=0.02)$ and a higher fraction of males $(14.2 \%$ vs. $8.4 \%, \mathrm{P}<0.001)$ than the 940 WADHA users who completed at least two online consultations.

\section{Discussion}

This longitudinal study is the first evaluation of the mHealth solution WADHA. The study found that $71 \%$ of the WADHA users exhibited weight loss. Furthermore, levels of PA increased while mood, quality of life, and body image satisfaction was improved and appetite, degree of bullying, and wish for weight loss was reduced.

Among the 940 WADHA users, the median BMI change was -0.63 BMI-points. The WADHA seems to replicate similar and significant reductions in BMI, as previously seen in original clinical studies of the HOT protocol $(7,10)$, indicating that the protocol can be transferred from children and adolescents to adult users and from clinical practice into an online format.

The reduction in BMI is also in accordance with a retrospective cohort study by Chin et al. where 35,921 adults followed a lifestyle behavioral changing app for a median of 267 days, resulting in a median BMI reduction of 1.7 and weight loss achieved in $77.9 \%$ of the app users (29). The lower BMI reduction and lower fraction with weight loss in the present study might be explained by the shorter treatment duration in the present study. In contrast, the present study reports larger weight loss fractions than observed in a large study by $\mathrm{Hu}$ et al. $(\mathrm{n}=8,977)$ that reported weight loss in $59 \%$ after $~ 340$ days of intervention, focusing solely on dietary habits, meal planning, recipes, and goal setting (30). The higher fraction of app users losing weight despite a shorter treatment duration in the present study could indicate that interventions addressing weight loss from multiple target points produce greater weight loss than the ones mainly focusing on dietary components. The present study found that $20 \%$ increased their BMI. Previous studies of the HOT protocol have shown that among children and adolescents who increased their BMI during treatment, 80\% improved their lipid concentrations and $61 \%$ improved their body composition despite the BMI increase (20). Measurements of body composition and lipid profile of the WADHA users would be of considerable interest, in order to investigate whether the WADHA can produce similar results.

A higher BMI at baseline was found to associate with greater reductions in $\mathrm{BMI}$ which is in accordance with the aforementioned studies of Chin et al. (29), Hu et al. (30), and a study of Hwang et al. (31) that evaluated a free online weight loss program (educational content, self-monitoring of weight, diet, exercise, and social support functions, $\mathrm{n}=1,258)$. All three studies similarly found that a higher $\mathrm{BMI}$ at baseline associated with greater weight loss.

A higher age was found to associate with greater reductions in BMI in the present study, which is in line with $\mathrm{Hu}$ et al. (30) who found the older users (older than 60 years) to lose more weight. In contrast, Chin et al. (29) found that younger users (younger than 33 years) lost more weight. The difference in results might be due to differences in target groups, advertisement platforms, price, and other commercial parameters. An evaluation of general medical practitioners in Denmark have found that patients above 65 years of age tend to engage more with physicians through e-mail consultations (32). It is possible, that the age distribution among the users in the present study could be explained by such a trend.

A greater weight loss was associated with a higher number of online consultations, which is in accordance with the aforementioned studies (29-31) reporting that more frequent use of an app was associated with greater weight loss.

Finally, male gender associated with greater reductions in BMI, which is in line with other studies $(29,30)$.

In the present study, the median number of days per week with PA for at least one hour per day increased by 1.5 days per week from 2.0 days per week at baseline. A 12-month randomized controlled trial by Thomas et al. (33) $(\mathrm{n}=279)$ compared (I) an online intervention (weight, diet, and exercise) with (II) the online intervention combined with a physical activity tracker, and (III) with a control group. They found a limited and temporary increase in PA of 1-7 minutes per day after three months and a decrease in PA of 1-2 minutes per day after 12 months in the intervention groups. Thomas et al. reported a five-percent weight loss in approximately $20 \%$ of the participants in the intervention groups. The greater fraction with weight loss in the present study could be explained by the configuration of an explicit, concrete, comprehensive, and tailor-made treatment plan in combination with a set of advices targeting many known aspects related to weight loss; all put together in 
one mHealth solution.

The WADHA users improved mood, quality of life, and body image satisfaction and reduced their appetite, bullying, and wish for weight loss. This improvement on all VAS qualities indicates that the WADHA users seem to experience a greater life fulfillment in general.

Psychosocial well-being has not been routinely investigated in regard to mHealth weight loss applications. However, a 24-week randomized controlled trial $(n=88)$ from Brindal et al. (34), comparing a 'control app' with tracking of weight, diet, and exercise to a 'full intervention app' including feedback and a possibility to evaluate on changes in mood, found an improved life satisfaction for both groups. These results are consistent with the present study in terms of reporting an improvement in mood and life-satisfaction. Mood and life satisfaction are found to be central in weight loss maintenance (35), and it would be interesting to investigate whether mood and life satisfaction could be used as indicators of long-term outcomes.

\section{Limitations}

Data from the present study have not been compared to a control group, which is an incorporate limitation arising from the study design. All data in this study were self-reported, which due to overestimation and social desirability, as well as typing errors and errors in measurement of height and weight, may have produced inaccurate data. Nevertheless, the degree of potential divergence associated with self-reported data are most likely the same at both baseline and follow-up, thus plausibly making the estimations of changes relevant and useful. It is remarkable that only $8.4 \%$ of the app users in the present study were males. In order to investigate the impact of gender more thoroughly, an equal distribution between genders is desirable.

Even though the HOT protocol primarily has been targeting children and adolescents, it has been shown to produce reductions in parents' BMI indicating a positive effect on the entire family (14). Therefore, it might be relevant to consider that the family as a whole might benefit from one WADHA household subscription-and that adult males consequently do not need to subscribe themselves, leading to a skewed gender distribution. Lastly, the relatively short study period is also a limitation, as a study spanning several years would be preferable in order to explore the long-term effects of the WADHA.

\section{Perspectives}

Mobile apps can be as effective as conventional weight loss programs, and the WADHA provides an alternative to inperson consultations, since the results are on par with those of a multifaceted, healthcare professional, hospital, and municipality setting $(7,10)$. A combination of conventional weight loss programs and an app-intervention have shown to be more effective (36). As the HOT Protocol and the mHealth app-version (WADHA) are based on the same treatment protocol, it would be interesting to investigate if this combination can offer an opportunity to further improve the treatment outcome for people living with obesity.

\section{Conclusions}

This longitudinal study shows that the results of an evidence-based, multifaceted obesity treatment model originally developed for in-person use in hospitals and primary care settings are reproducible in an online-only version. The study showed weight loss in $71.4 \%$ of the WADHA users, increase in PA levels, improvement of mood, quality of life, and body image satisfaction, and reduction in appetite, degree of bullying, and wish for weight loss. Furthermore, this study showed that we are capable of transferring a protocol designed for children and adolescents to adults.

\section{Acknowledgments}

Funding: None.

\section{Footnote}

Reporting Checklist: The authors have completed the STROBE checklist. Available at https://dx.doi. org/10.21037/mhealth-21-8

Data Sharing Statement: Available at https://dx.doi. org $/ 10.21037 / \mathrm{mhealth}-21-8$

Peer Review File: Available at https://dx.doi.org/10.21037/ mhealth-21-8

Conflicts of Interest: All authors have completed the ICMJE uniform disclosure form (available at https://dx.doi. org/10.21037/mhealth-21-8). CEF reports stock or stock 
options in Dr Holm group. JCH reports ownership of Dr Holm Group and serves as an advisor of The Novo Nordisk Global Childhood Obesity advisory board, Advisor and lecturer of Rhythm Pharmaceuticals, advisor and assessor of German Centre for Child and Youth Health, and Executive member of European Association for the Study of Obesity (EASO). AFP declares no conflict of interest, but JCH is his father-in-law. The other authors have no conflicts of interest to declare.

Ethical Statement: The authors are accountable for all aspects of the work in the ensuring that questions related to the accuracy or integrity of any part of the work are appropriately investigated and resolved. All included data from the study population is given with consent, and WADHA users have consented to having their data analyzed and stored according to the European GDPR regulations. This study was conducted according to ethical standards defined by the Danish national research ethics committees and the Helsinki Declaration (as revised in 2013). In accordance with Danish Law (section 14(2) of the Committee Act of the Danish National Committee on Health Research Ethics), this questionnaire-based survey study, which does not involve human biological material, should not be notified or reported to a research ethics committee.

Open Access Statement: This is an Open Access article distributed in accordance with the Creative Commons Attribution-NonCommercial-NoDerivs 4.0 International License (CC BY-NC-ND 4.0), which permits the noncommercial replication and distribution of the article with the strict proviso that no changes or edits are made and the original work is properly cited (including links to both the formal publication through the relevant DOI and the license). See: https://creativecommons.org/licenses/by-nc-nd/4.0/.

\section{References}

1. Obesity and overweight. Accessed 22/11 2019. Available online: https://www.who.int/en/news-room/fact-sheets/ detail/obesity-and-overweight

2. Kelly T, Yang W, Chen CS, et al. Global burden of obesity in 2005 and projections to 2030. Int J Obes (Lond) 2008;32:1431-7.

3. Williams EP, Mesidor M, Winters K, et al. Overweight and Obesity: Prevalence, Consequences, and Causes of a Growing Public Health Problem. Curr Obes Rep
2015;4:363-70.

4. Subhi Y, Bube SH, Rolskov Bojsen S, et al. Expert Involvement and Adherence to Medical Evidence in Medical Mobile Phone Apps: A Systematic Review. JMIR Mhealth Uhealth 2015;3:e79.

5. Chen J, Cade JE, Allman-Farinelli M. The Most Popular Smartphone Apps for Weight Loss: A Quality Assessment. JMIR Mhealth Uhealth 2015;3:e104.

6. Holzmann SL, Holzapfel C. A Scientific Overview of Smartphone Applications and Electronic Devices for Weight Management in Adults. J Pers Med 2019;9:31.

7. Holm JC, Gamborg M, Bille DS, et al. Chronic care treatment of obese children and adolescents. Int J Pediatr Obes 2011;6:188-96.

8. Barlow SE; Expert Committee. Expert committee recommendations regarding the prevention, assessment, and treatment of child and adolescent overweight and obesity: summary report. Pediatrics 2007;120 Suppl 4:S164-92.

9. Schwartz MW, Seeley RJ, Zeltser LM, et al. Obesity Pathogenesis: An Endocrine Society Scientific Statement. Endocr Rev 2017;38:267-96.

10. Most SW, Højgaard B, Teilmann G, et al. Adoption of the children's obesity clinic's treatment (TCOCT) protocol into another Danish pediatric obesity treatment clinic. BMC Pediatr 2015;15:13.

11. Kloppenborg JT, Gamborg M, Fonvig CE, et al. The effect of impaired glucose metabolism on weight loss in multidisciplinary childhood obesity treatment. Pediatr Diabetes 2018;19:366-74.

12. Hollensted M, Fogh M, Schnurr TM, et al. Genetic Susceptibility for Childhood BMI has no Impact on Weight Loss Following Lifestyle Intervention in Danish Children. Obesity (Silver Spring) 2018;26:1915-22.

13. Fogh M, Lund MAV, Mollerup PM, et al. Disturbed eating behaviours do not impact treatment response in a paediatric obesity chronic care treatment programme. J Paediatr Child Health 2020;56:542-9.

14. Trier C, Dahl M, Stjernholm T, et al. Effects of a FamilyBased Childhood Obesity Treatment Program on Parental Weight Status. PLoS One 2016;11:e0161921.

15. Mollerup PM, Gamborg M, Trier C, et al. A hospitalbased child and adolescent overweight and obesity treatment protocol transferred into a community healthcare setting. PLoS One 2017;12:e0173033.

16. Mollerup PM, Lausten-Thomsen U, Fonvig CE, et al. Reductions in blood pressure during a communitybased overweight and obesity treatment in children and 
adolescents with prehypertension and hypertension. J Hum Hypertens 2017;31:640-6.

17. Hvidt KN, Olsen MH, Ibsen H, et al. Effect of changes in BMI and waist circumference on ambulatory blood pressure in obese children and adolescents. J Hypertens 2014;32:1470-7; discussion 1477.

18. Hvidt KN, Olsen MH, Ibsen H, et al. Weight reduction and aortic stiffness in obese children and adolescents: a 1-year follow-up study. J Hum Hypertens 2015;29:535-40.

19. Nielsen TR, Gamborg M, Fonvig CE, et al. Changes in lipidemia during chronic care treatment of childhood obesity. Child Obes 2012;8:533-41.

20. Nielsen TRH, Fonvig CE, Dahl M, et al. Childhood obesity treatment; Effects on BMI SDS, body composition, and fasting plasma lipid concentrations. PLoS One 2018;13:e0190576.

21. Fonvig CE, Chabanova E, Ohrt JD, et al. Multidisciplinary care of obese children and adolescents for one year reduces ectopic fat content in liver and skeletal muscle. BMC Pediatr 2015;15:196.

22. Andersen IG, Holm JC, Homøe P. Impact of weight-loss management on children and adolescents with obesity and obstructive sleep apnea. Int J Pediatr Otorhinolaryngol 2019;123:57-62.

23. Mollerup PM, Nielsen TRH, Bøjsøe C, et al. Quality of life improves in children and adolescents during a community-based overweight and obesity treatment. Qual Life Res 2017;26:1597-608.

24. Fonvig CE, Hamann SA, Nielsen TRH, et al. Subjective evaluation of psychosocial well-being in children and youths with overweight or obesity: the impact of multidisciplinary obesity treatment. Qual Life Res 2017;26:3279-88.

25. DrHolmApp.dk. Accessed 23/4 2021. Available online: https://drholmapp.dk

26. DrHolmApp.co.uk. Accessed 23/4 2021. Available online: http://www.drholmapp.co.uk

doi: $10.21037 /$ mhealth-21-8

Cite this article as: Langkjær IOJ, Fonvig CE, Holm LA, Pihl AF, Holm JC. Longitudinal evaluation of an mHealth overweight and obesity management tool. mHealth 2022;8:2.
27. Processing of data when subscribing to DrHolmApp. com. Accessed 22/11 2019. Available online: https://www. jenschristianholm.dk/uk/gdpr/subscribing-to-drholmapp.. aspx

28. R Core Team 2015. R: A language and environment for statistical computing. R Foundation for Statistical Computing, Vienna, Austria. Available online: https:// www.R-project.org/

29. Chin SO, Keum C, Woo J, et al. Successful weight reduction and maintenance by using a smartphone application in those with overweight and obesity. Sci Rep 2016;6:34563.

30. Hu EA, Nguyen V, Langheier J, et al. Weight Reduction Through a Digital Nutrition and Food Purchasing Platform Among Users With Obesity: Longitudinal Study. J Med Internet Res 2020;22:e19634.

31. Hwang KO, Ning J, Trickey AW, et al. Website usage and weight loss in a free commercial online weight loss program: retrospective cohort study. J Med Internet Res 2013;15:e11.

32. Praktiserende Lægers Organisation. PLO faktaark. 2020. Available online: https://www.laeger.dk/sites/ default/files/plo_faktaark_2021_juni_2021.pdf

33. Thomas JG, Raynor HA, Bond DS, et al. Weight loss in Weight Watchers Online with and without an activity tracking device compared to control: A randomized trial. Obesity (Silver Spring) 2017;25:1014-21.

34. Brindal E, Hendrie GA, Freyne J, et al. A Mobile Phone App Designed to Support Weight Loss Maintenance and Well-Being (MotiMate): Randomized Controlled Trial. JMIR Mhealth Uhealth 2019;7:e12882.

35. Robertson S, Davies M, Winefield H. Positive psychological correlates of successful weight maintenance in Australia. Clin Psychol 2017;21:236-44.

36. Ahn JS, Lee H, Kim J, et al. Use of a Smartphone App for Weight Loss Versus a Paper-Based Dietary Diary in Overweight Adults: Randomized Controlled Trial. JMIR Mhealth Uhealth 2020;8:e14013. 


\title{
Appendix 1 Example of a treatment plan
}

The treatment plan is personal, and the following example is based on a single person's answers. The treatment plan can therefore contain less, more, or different treatment plan points depending on individual answers.

\author{
Personal treatment plan \\ $\square$ 1. Be less picky. \\ $\square$ 2. Eat breakfast every day. \\ 3. Breakfast: not biscuits
}

$\square$ 4. Breakfast: You can freely choose between oats, rolled oats and oatmeal porridge with a maximum of one teaspoon of raisins/sugar, puffed rye or oat grains (not sweetened), plain yogurt with a maximum of $0.5 \%$ fat content, greens, rye bread with lean toppings/cold cuts with a maximum of $9 \%$ fat and cheese with a maximum of 13 $\%$ fat.

$\square 5$. For lunch, choose freely between greens, rye bread with lean toppings/cold cuts with a maximum of $9 \%$ fat and cheese with a maximum of $13 \%$ fat.

$\square 6$. Eat an afternoon meal every day (between lunch and dinner).

$\square 7$. Afternoon meal: not coarse bread, Frosties, puffed wheat grains, whole milk, white pasta or coffee / tea with sugar, honey or sweetener.

$\square$ 8. Afternoon meal: just like breakfast, you can freely choose between oats, rolled oats and oatmeal porridge with a maximum of one teaspoon of raisins/sugar, puffed rye or oat grains (not sweetened), plain yogurt with a maximum of $0.5 \%$ fat content, greens, rye bread with lean toppings/cold cuts with a maximum of $9 \%$ fat and cheese with a maximum of $13 \%$ fat.

$\square$ 9. Eat dinner every day.

$\square$ 10. Dinner: not crispbread, cheese with more than $13 \%$ fat or whole milk.

$\square$ 11. Dinner: eat a mixed selection of greens. Choose freely between potatoes, whole-grain pasta, brown rice and rye bread. Choose freely between lean meats without visible fat, fish (both lean and fat) and legumes. Minced meat must contain no more than $7 \%$ fat. A maximum of 2 tablespoons of dressing or sauce per serving. Use as little fat as possible and a maximum of 2 teaspoons per person for cooking warm food.

$\square$ 12. Dinner must be portioned (arranged on a plate) in the kitchen.

$\square$ 13. Dinner must be portioned according to the T-plate model.

$\square$ 14. Dinner: if a 2nd portion (2nd serving) is needed, it may be served no sooner than 20 minutes after the 1 st portion is finished.

$\square$ 15. Late-night meal: Do not eat between dinner and breakfast. But if you are hungry, you can choose from 1/2-2 slices of rye bread (possibly roasted) with toppings/cold cuts with a maximum of $9 \%$ fat content and cheese with a maximum of $13 \%$ fat content, greens, oats, rolled oats, oatmeal porridge, puffed rye or oat grains (not sweetened), and plain yogurt with a maximum of $0.5 \%$ fat content.

$\square$ 16. Late-night meal: not biscuits or white rice between dinner and breakfast.

$\square$ 17. You must feel satiated (full) - but not over satiated - after each of the main meals: breakfast, lunch, the afternoon meal and dinner.

$\square$ 18. Salad and vegetables: eat vegetables every day.

$\square$ 19. Choose as little oil or fat as possible when cooking, putting spreads on bread or purchasing dairy products.

$\square$ 20. Fast food: maximum once a month.

$\square$ 21. Dried fruits and nuts: maximum once a week, equivalent to one small handful (30-40 gram) of dried fruits and nuts per week.

22. Milk: maximum 0.5 liters of dairy products per day, with a maximum fat content of $0.5 \%$.

$\square$ 23. Sweets/candy and snacking: maximum once a week. See how much under your empty-calories-allowance.

$\square$ 24. Reduce your alcohol intake to a maximum of 3 units of alcohol per week.

$\square$ 25. Physical activity: be physically active for at least 1 hour every day, where you can feel the pulse rising and your breathing getting faster.

$\square 26$. Walk or take the bike as much as possible for your everyday transport.

$\square 27$. It is important that you quit smoking.

You can check the boxes in the treatment plan as you feel you are managing them.

Blue words in the treatment plans are active links to the library with thorough explanations readily available. All aspects of the use of the WADHA are subject to personal support within the interactive communication hub. Boxes reflect interactive participation with the WADHA user so that she or he can tick treatment plan points to provide an overview of what is achieved and what is lacking within the personalized treatment plan. For example, box number 3 have been ticked off and is thus achieved in treatment. It is now shown with a light grey font appearing in the background, though still readable. 